Corrigendum

\title{
Corrigendum to "Designing a Human Machine Interface for Quality Assurance in Car Manufacturing: An Attempt to Address the "Functionality versus User Experience Contradiction" in Professional Production Environments"
}

\author{
Nikolaj Borisov $\mathbb{D},{ }^{1}$ Benjamin Weyers $\mathbb{D}^{2},{ }^{2}$ and Annette Kluge $\mathbb{D}^{3}$ \\ ${ }^{1}$ University of Duisburg-Essen, 47057 Duisburg, Germany \\ ${ }^{2}$ Computer Science Department, RWTH Aachen University, 52074 Aachen, Germany \\ ${ }^{3}$ Department of Psychology, Ruhr-University Bochum, 44801 Bochum, Germany \\ Correspondence should be addressed to Benjamin Weyers; weyers@vr.rwth-aachen.de \\ Received 16 April 2019; Accepted 21 April 2019; Published 12 June 2019 \\ Copyright (C) 2019 Nikolaj Borisov et al. This is an open access article distributed under the Creative Commons Attribution License, \\ which permits unrestricted use, distribution, and reproduction in any medium, provided the original work is properly cited.
}

In the article titled "Designing a Human Machine Interface for Quality Assurance in Car Manufacturing: An Attempt to Address the "Functionality versus User Experience Contradiction" in Professional Production Environments" [1], there was an error in Table 4 during the editing process as the reported values are wrongly sorted in the table. However, they are correct in the text. Accordingly, the change did not affect the results of the article. The correct version of Table 4 should be as follows: 
TABLE 4: Device score model: results overview (the lower the better).

\begin{tabular}{lcccccccc}
\hline DEVICE SCORE MODEL & & Smartphone & Tablet & Headset & AR-Mic. & AR-Gest. & Proj.-Mic. & Proj.-Gest. \\
\hline \multicolumn{1}{c}{ Overall grade } & & 2,1 & 2,2 & 3,0 & 2,6 & 3,5 & 3,6 & 4,2 \\
\hline Criterion & Weight. & Grade & Grade & Grade & Grade & Grade & Grade & Grade \\
\hline Ergonomics & $25 \%$ & 2,4 & 3,4 & 3,0 & 3,2 & 3,2 & 3,1 & 2,9 \\
Performance & $40 \%$ & 1,7 & 1,9 & 2,8 & 1,7 & 2,9 & 4,4 & 5,8 \\
Technology acceptance & $15 \%$ & 1,7 & 1,1 & 5,0 & 3,3 & 2,0 & 5,1 & 4,4 \\
User Experience & $20 \%$ & 2,7 & 2,3 & 1,8 & 3,2 & 6,0 & 1,7 & 2,6 \\
\hline
\end{tabular}

AR-Mic $=$ augmented reality: data glasses with microphone as input device.

AR-Gest. = augmented reality: data glasses with hand gesture as input device.

Proj.-Mic. $=$ display instructions over projection with microphone as input device.

Proj.-Gest. = display instructions over projection with hand gesture as input.

\section{References}

[1] N. Borisov, B. Weyers, and A. Kluge, "Designing a human machine interface for quality assurance in car manufacturing: an attempt to address the "functionality versus user experience contradiction" in professional production environments," Advances in Human Computer Interaction, vol. 2018, Article ID 9502692, 18 pages, 2018. 


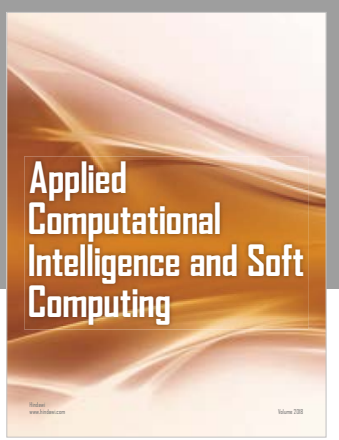

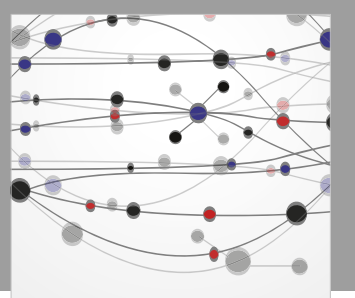

The Scientific World Journal
Submit your manuscripts at

Computing
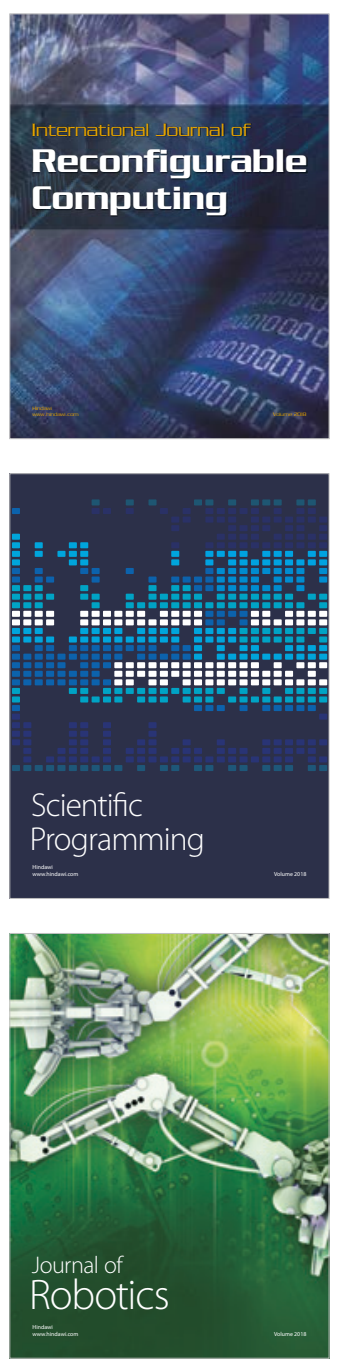

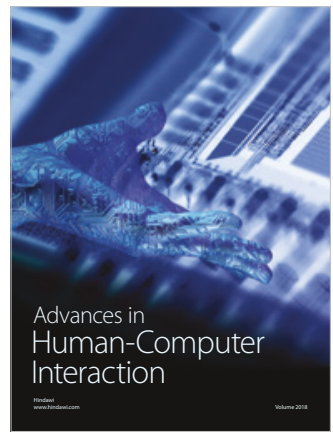

Human-Compute

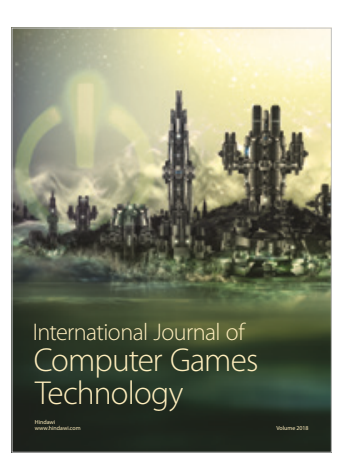

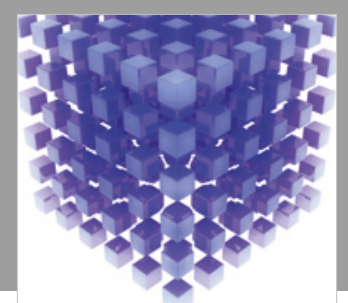

Mathematical Problems in Engineering

\section{Engincering}
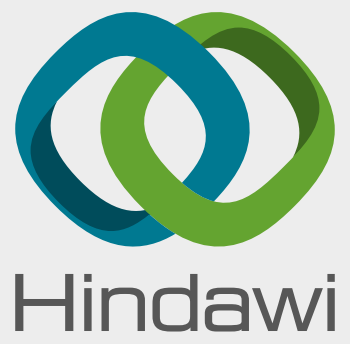

www.hindawi.com
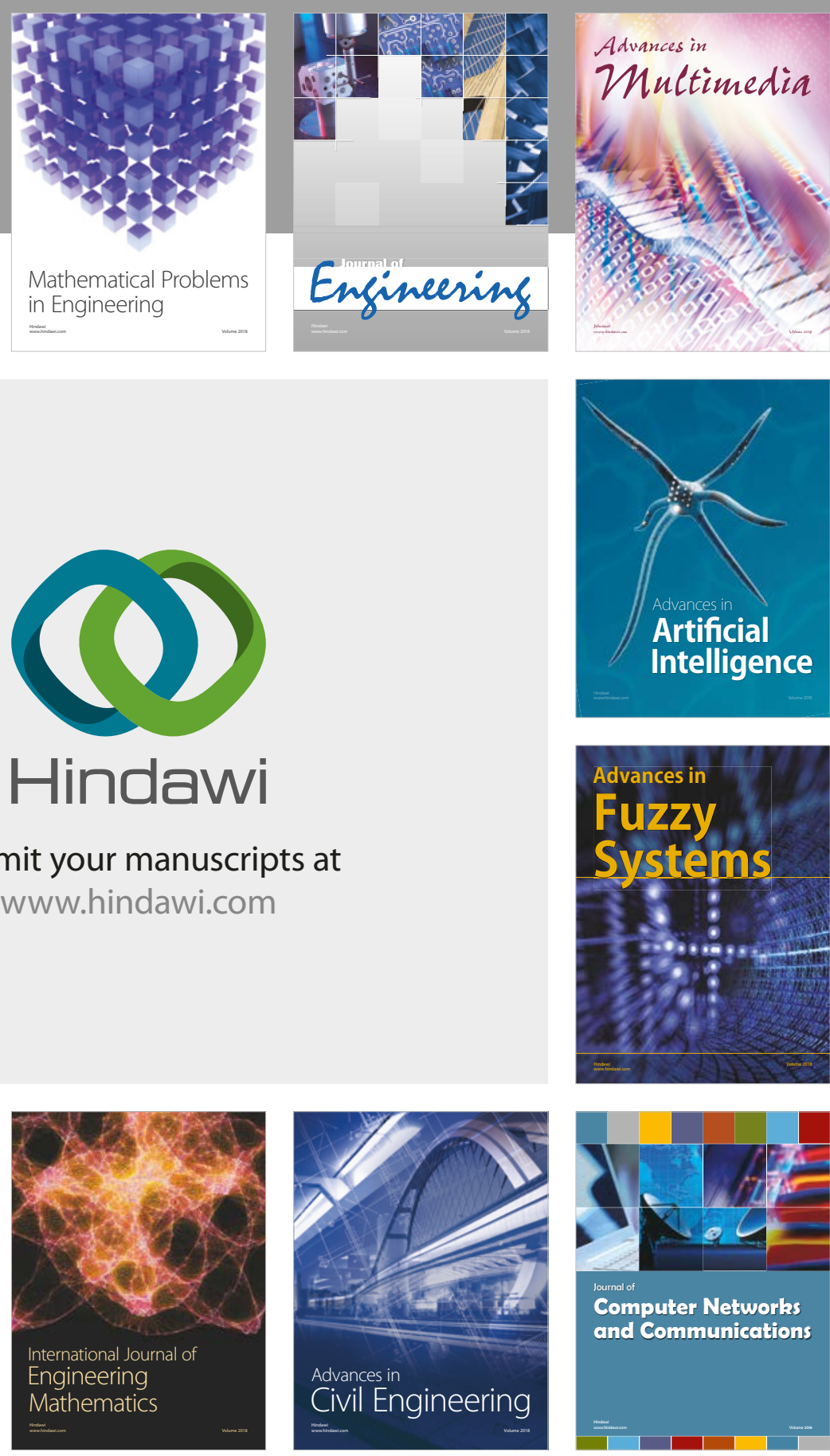

Computer Networks and Communications

Multimedia
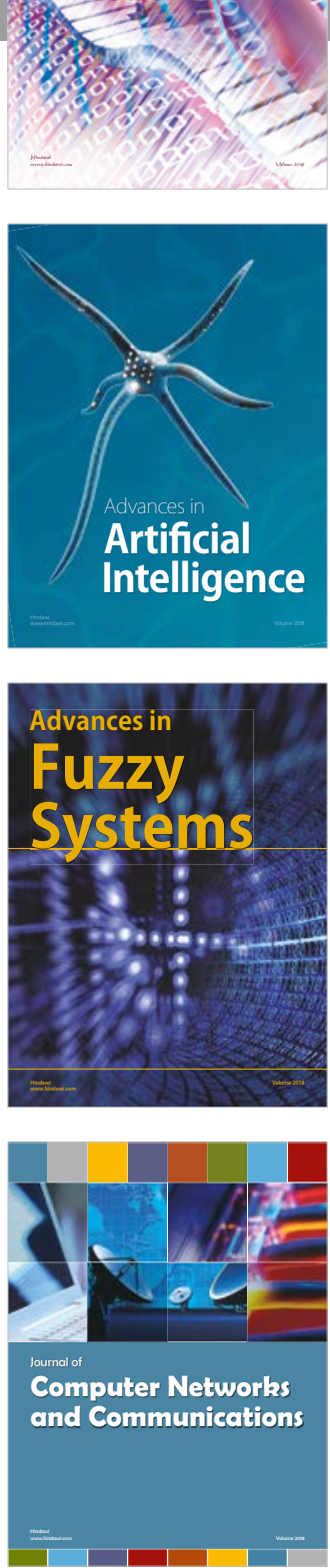

Advances in

Modelling \&

Simulation

in Engineering

interaction

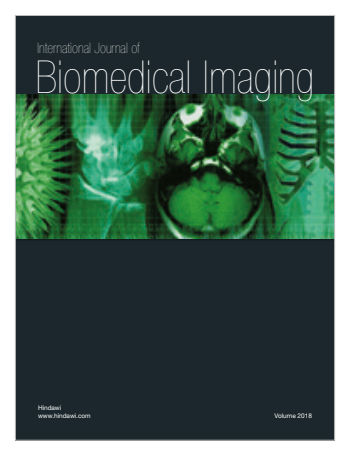

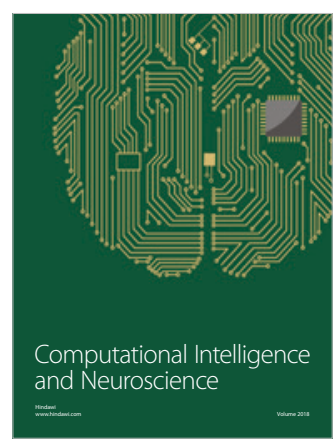

